\title{
Enzymatic reactions in teleocidin B biosynthesis
}

\author{
Takayoshi Awakawa ${ }^{1,2} \mathbb{E}$
}

Received: 17 January 2021 / Accepted: 1 March 2021 / Published online: 6 March 2021

(c) The Author(s) 2021

\begin{abstract}
The teleocidin B family members are terpene indole compounds isolated from Streptomyces bacteria, and they strongly activate protein kinase $\mathrm{C}$ (PKC). Their unique structures have attracted many researchers in the natural product chemistry and pharmacology fields, and numerous isolation and bioactivity studies have been conducted. The accumulated information has facilitated the identification of the enzymatic reactions in teleocidin biosynthesis, and new developments in structural biology have strongly aided efforts to clarify the finer points of these reactions. This review describes the recent biochemical and structural biological studies to reveal their reaction mechanisms, with a primary focus on the terpene cyclization triggered by the $\mathrm{C}-\mathrm{N}$ bond formation by $\mathrm{P} 450$ oxygenase (TleB), the prenyltransferase (TleC), and the methyltransferase (TleD). This new knowledge will benefit future engineering studies to create unnatural PKC activators.
\end{abstract}

Keywords Terpene indole $\cdot$ Biosynthesis $\cdot$ Protein kinase $\mathrm{C} \cdot \mathrm{X}$-ray crystal structure analysis

\section{Introduction}

Terpene indole compounds have an abundance of structural diversity, derived from the electron-rich indole ring and the cation-generating terpenoid [1-5]. This group includes large numbers of bioactive compounds, including ergotamine (vasoconstrictor), vinblastine (microtubule dynamics inhibitor), spirotryprostatin (antimitotic arrest agent), and teleocidin $\mathrm{B}$ (protein kinase $\mathrm{C}$ activator) (Fig. 1). The unique structure of teleocidin $\mathrm{B}$ and its robust bioactivity in the activation of protein kinase $\mathrm{C}$ (PKC) have drawn keen attention from natural product researchers [6]. Numerous teleocidin B analogs have been isolated from natural sources (Fig. 2) since the first compound teleocidin B-4 was identified through NMR and X-ray structural studies [7-10]. The isolated analogs not only furnished a pool of natural medicinal compounds but also provided beneficial information to construct the hypothetical biosynthetic pathways. In addition, isotope-feeding and chemical transformation

Takayoshi Awakawa

awakawa@mol.f.u-tokyo.ac.jp

1 Graduate School of Pharmaceutical Sciences, The University of Tokyo, Bunkyo-ku, Tokyo 113-0033, Japan

2 Collaborative Research Institute for Innovative Microbiology, The University of Tokyo, Yayoi 1-1-1, Bunkyo-ku, Tokyo 113-8657, Japan experiments have generated useful clues for deducing biosynthetic pathways, such as the transformation of blastmycetin D to olivoretin A by acid treatment $[11,12]$. The isolation of lyngbyatoxin A (=teleocidin A-1) from the cyanobacterium Moorea producens [13], and the establishment of its biosynthetic gene cluster also facilitated the identification of the teleocidin gene cluster [14]. The PKC activators are recognized as tumor growth enhancers, but a recent study has reported that the subtype-specific activation of PKC actually represses tumor growth [15]. Thus, pharmacologically useful analogs are likely to be present in the library of teleocidin analogs. Therefore, we investigated the biosynthesis of teleocidin for the biosynthetic construction of a base for the creation of a library of PKC activators. This review updates the prior reviews on teleocidin biosynthesis [16, 17], and mainly describes the discovery of the gene cluster and the biochemical and structural studies of the biosynthetic enzymes in teleocidin biosynthesis.

\section{Identification of teleocidin biosynthetic gene cluster (tle cluster)}

The biosynthetic gene cluster of teleocidin B (tle cluster), which includes genes encoding a non-ribosomal peptide synthetase (NRPS, tleA), P450 oxygenase (tleB), and prenyltransferase $(t l e C)$, was found in the genome of a teleocidin 
Fig. 1 Structures of representative terpene indole alkaloids
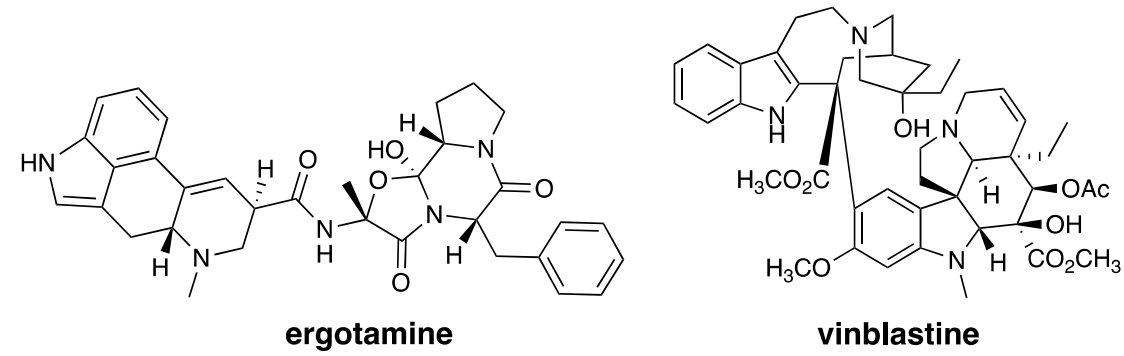<smiles>COc1ccc2c(c1)NC(=O)[C@]21C[C@H]2C(=O)N3CCC[C@H]3C(=O)N2[C@H]1C=C(C)C</smiles>

spirotryprostatin A teleocidin B<smiles></smiles>

B-1 (19R, 25S

B-2 (19S, 25R)

B-3 (19S, 25S)

B-4 (19R, 25R)

Fig. 2 Structures of the isolated teleocidin compounds

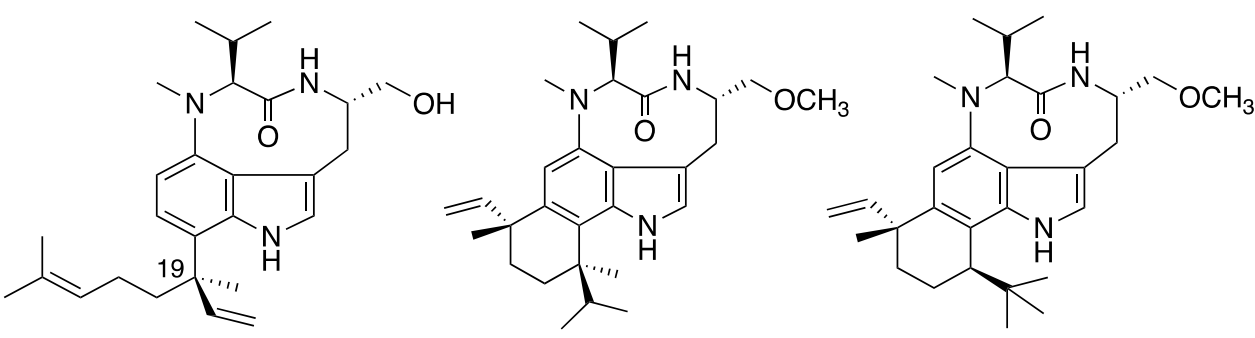

olivoretin C olivoretin E

= lyngbyatoxin A<smiles>C=C[C@H](CCC(C)(O)C(C)C)c1ccc(N(C)[C@@H](C(=O)N[C@@H](CC)CO)C(C)C)c2[nH]cc(CC(C)C)c12</smiles>

blastmycetin D<smiles></smiles>

olivoretin A
B producer, Streptomyces blastmyceticus, based on the similarities to lyngbyatoxin biosynthetic enzymes in cyanobacteria [18]. The reactions of TleABC were deduced from the lyngbyatoxin biosynthetic reactions $[14,19,20]$, as the synthesis of $N$-methyl-L-valyl-L-tryptophanol (NMVT) by TleA, the C-N bond formation by TleB, and the prenyl transfer by TleC (Fig. 3). We expected that the 23-kb genomic region including the tle cluster would be responsible for the production of teleocidin B. Unexpectedly, the heterologous expression of the tle cluster in Streptomyces lividans yielded only teleocidin A-1 (Fig. 2). Thus, the C-methyltransferase (C-MT) genes encoded outside of the tle cluster were screened by co-expressing C-MT genes in addition to the tle cluster. As a result, the expression of one of the transcribed C-MT genes (named tleD) led to the production of teleocidin B-1, teleocidin B-4, and des-O-methylolivoretin $\mathrm{C}$ (Fig. 4), indicating that these compounds are derived from a common intermediate and the terpene structure diversifies after $\mathrm{C}$-methylation. The in vitro TleD reaction with [D-25]lyngbyatoxin A and $S$-adenosylmethionine (SAM) produced [D-26]teleocidin B-4, suggesting that the deuterium atom shifts from $\mathrm{C}-25$ to $\mathrm{C}-26$ during the terpene ring cyclization. Finally, we proposed the reaction of TleD: first, C-25 was C-methylated from SAM by TleD, and the resultant cation migrated from C-25 to C-26. Secondly, the cation at 
Fig. 3 Biosynthetic reactions of lyngbyatoxin $\mathrm{A}=$ teleocidin $\mathrm{A}-1$
Fig. 4 Terpene cyclization triggered by the methylation of teleocidin A- 1 by TleD

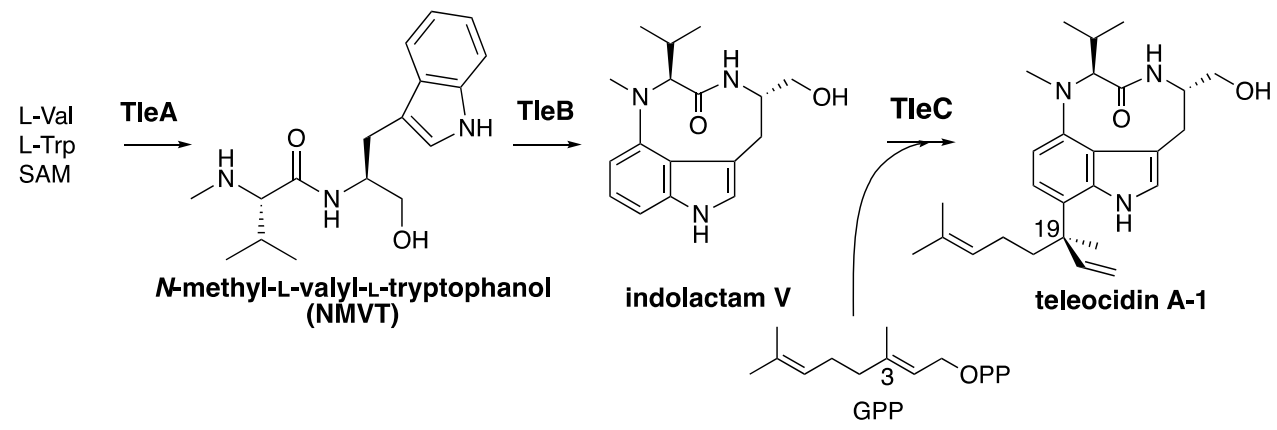

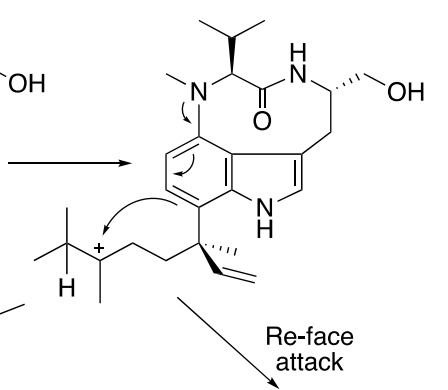

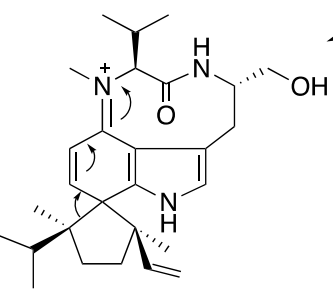

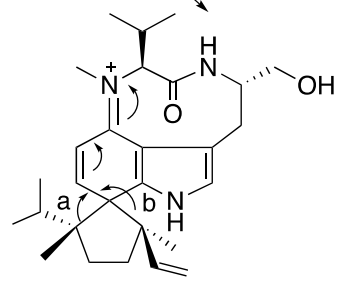

path a<smiles>CCC</smiles>

teleocidin B-1<smiles></smiles>

teleocidin B-1<smiles>C=C[C@]1(C)CC[C@@](C)(C(C)C)c2cc(N(C)C(C(=O)N[C@H](CO)C(C)C)C(=O)N[C@H](C)C(C)C)c3cc[nH]c3c21</smiles><smiles>C=C[C@]1(C)CC[C@](C)(C(C)C)C2=C1C=C(N(C)C(C(=O)N[C@@H](CC)CO)C(C)C)c1cc2c[nH]1</smiles>

des-O-methylolivoretin $\mathrm{C}$
C-25 reacted with C-7 of the indole in $R e$-face attack to form the spiro intermediate. The spiro intermediate spontaneously transformed into teleocidin B-4 via path A and des- $O$-methylolivoretin $\mathrm{C}$ via path B (Fig. 4). The unfavored $\mathrm{Si}$-face attack yielded the spiro intermediate with differently oriented vinyl and isopropyl groups, leading to the minor product, teleocidin B-1. This was the first identification of terpene cyclization triggered by $\mathrm{C}$-methylation in nature.

\section{Structural analysis of TleB}

The nine-membered indolactam $\mathrm{V}$ structure is the most important for PKC activation in teleocidin B [21], and its $\mathrm{C}-\mathrm{N}$ bond is constructed by a P450 monooxygenase which is a versatile heme-iron protein [22]. A P450 oxygenase encoded in the tle cluster, TleB, was deduced to accept 
NMVT and form the C-N bond between N-13 and C-4 to produce indolactam $\mathrm{V}$, as shown in the LtxB reaction [20]. Although the reaction mechanism of the $\mathrm{C}-\mathrm{N}$ bond formation was previously discussed in the literature [20, 23], the molecular basis and reaction mechanism of TleB/ LtxB have not been identified. Thus, we conducted biochemical analyses with substrate analogs and X-ray crystal structure analyses of TleB and HinD, a protein homolog with 57.5\% identity from Streptoalloteichus hindustanus, to clarify the reaction mechanism of this unique $\mathrm{C}(\mathrm{sp} 2)-\mathrm{H}$ amination and create unnatural indolactams [24]. When we utilized NMVT or $N$-methyl-L-phenylalanyl-L-tryptophanol (NMFT) as a substrate, TleB accepted only NMVT to produce 1 (the label in reference 24) and HinD accepted both NMVT and NMFT to produce indolactams 1 and 4 (Fig. 5). Next, the analogs with $\mathrm{N}-13$ substituted with $-\mathrm{NH}_{2}(\mathbf{5}),-\mathrm{OCH}_{3}(\mathbf{6}, \mathbf{1 1})$, or $-\mathrm{OH}(\mathbf{7}, \mathbf{1 2})$ and those with indole ring substitutions with benzo $[b]$ thiophene $(\mathbf{1 3}$, 14) and $N 1$-methyl indole $(\mathbf{1 5}, \mathbf{1 6})$ were tested (Fig. 5). Interestingly, TleB accepted 5-7 and yielded 6/5/6 tricyclic ring compounds $(\mathbf{8}-\mathbf{1 0})$, which are generated through the reaction between $14-\mathrm{OH}$ and $\mathrm{C}-2$ via the epoxidation between $\mathrm{C} 1-\mathrm{C} 2$. In addition to 9 , TleB also generated the demethylated product 7 from $\mathbf{6}$. HinD exhibited broader substrate specificity and oxidized 5-7, 11, and 12 to produce the tricyclic products $\mathbf{8 - 1 0}, \mathbf{1 7}$, and $\mathbf{1 8}$ and the demethylated products $\mathbf{7}$ and $\mathbf{1 2}$. HinD also produced the $O$-demethylated products 19 and $\mathbf{2 0}$ from the benzo[ $b$ ]thiophene substrates 13 and $\mathbf{1 4}$, and the $N$-demethylated products 21 and 22 from the N1-methylated substrates 15 and 16, respectively. These results indicated that $\mathrm{N}-13$ and $\mathrm{N}-1$ should react with the ferryl oxo species $\left(\mathrm{Fe}^{\mathrm{IV}}=\mathrm{O}\right)$ in $\mathrm{TleB}$, and their hydrogen atoms should be abstracted to generate the diradical. The TleB structure complexed with NMVT (1.90 A resolution) and the HinD structure complexed with 13 were solved by X-ray structural analyses $(2.35 \AA$ resolution) [24]. Their overall structures adopt the trigonal prism-fold consistently with the $\mathrm{P} 450$ oxygenases [25]. In the TleB complex structure, the N-1 of NMVT was closest to the heme iron (5.1 $\AA$ ), while the $\mathrm{N}-13$ of $\mathbf{1 3}$ was closest in the HinD complex structure (4.3 $\AA$ ) (Fig. 6). These data suggested that the hydrogen atom of NH-1 should be abstracted to generate the first radical and the NH-13 is then abstracted to generate the second radical. When an NMFT analog in which the N-methyl group is substituted with a cyclopropyl group was used as a substrate, we detected the product with $\mathrm{NH}_{2}-13$ generated after the reaction of the radical on $\mathrm{N}-13$ with the cyclopropane ring. This result reinforced the hypothesis that the hydrogen atom is abstracted from $\mathrm{N}-13$.
Fig. 5 The reactions of TleB and HinD
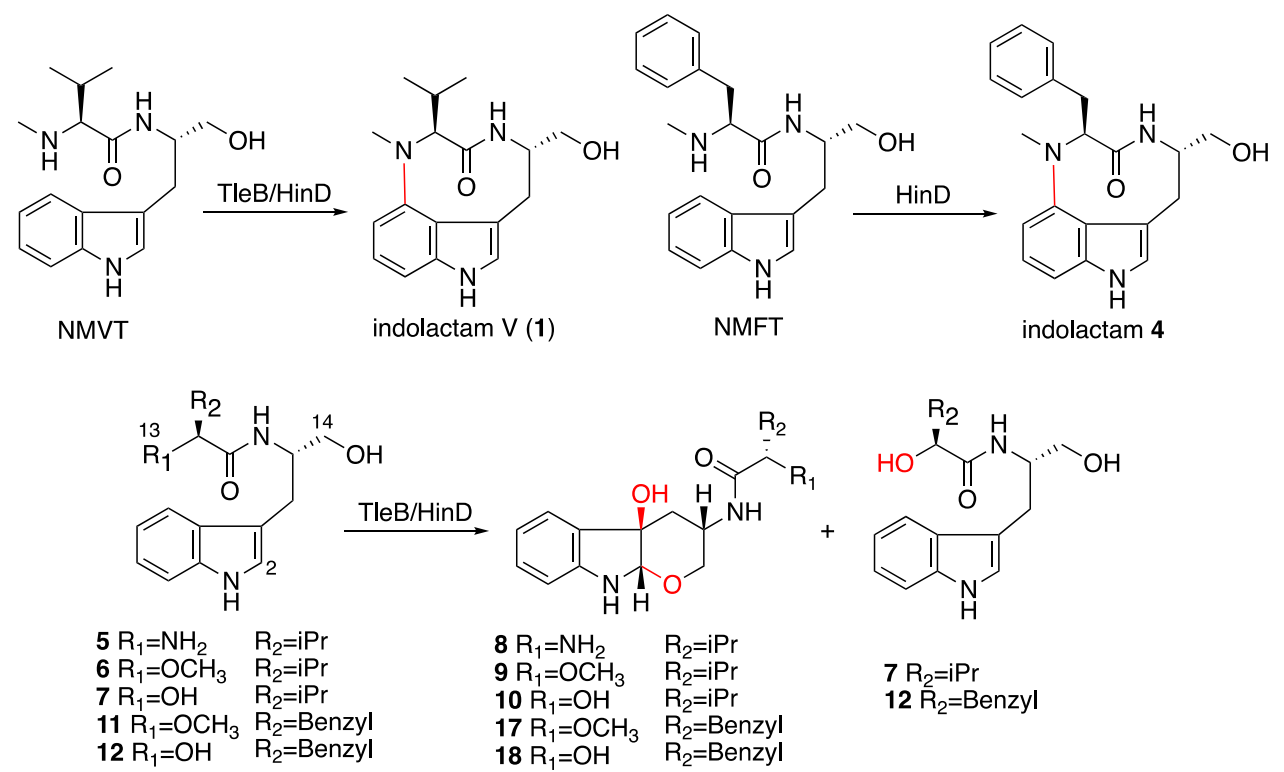

$\begin{array}{ll}8 \mathrm{R}_{1}=\mathrm{NH}_{2} & \mathrm{R}_{2}=\mathrm{iPr} \\ 9 \mathrm{R}_{1}=\mathrm{OCH}_{3} & \mathrm{R}_{2}=\mathrm{iPr}\end{array}$ $10 \mathrm{R}_{1}=\mathrm{OH}^{3} \quad \mathrm{R}_{2}=\mathrm{iPr}$

$17 \mathrm{R}_{1}=\mathrm{OCH}_{3} \quad \mathrm{R}_{2}=$ Benzyl

$18 \mathrm{R}_{1}=\mathrm{OH} \quad \mathrm{R}_{2}=$ Benzyl

$7 \mathrm{R}_{2}=\mathrm{iPr}$

$12 \mathrm{R}_{2}=$ Benzyl

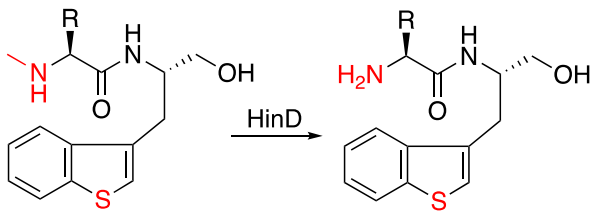

$13 \mathrm{R}=\mathrm{iPr}$ $14 \mathrm{R}=$ Benzyl

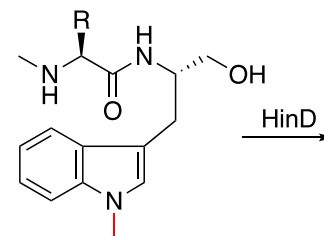

$15 \mathrm{R}=\mathrm{iPr}$ 16 R=Benzy<smiles>[R7]C(NC)C(=O)N[C@@H](CO)Cc1cn(CO)c2ccccc12</smiles> 
Fig. 6 The active site structures of TleB (brown, complexed with NMVT, PDB: 6J83) and HinD (cyan, complexed with the benzo[b]thiophene 13, PDB: 6J88)

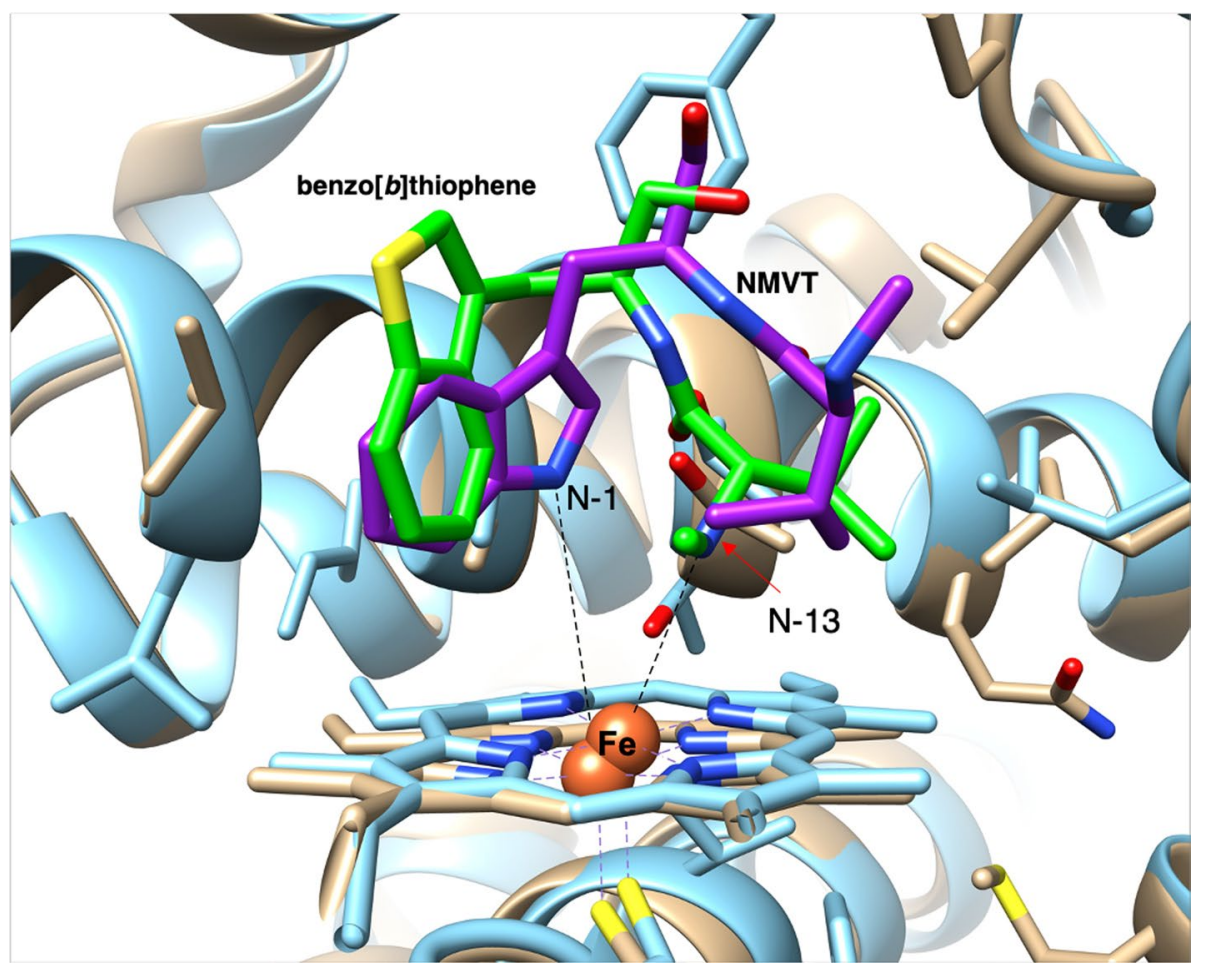

The data shown above supported the diradical pathway in which the $\mathrm{P} 450$ generates two radicals from NH-1 and $\mathrm{NH}-13$, and the following radical coupling forms the $\mathrm{C}-\mathrm{N}$ bond. Firstly, the ferryl oxo species abstracts $\mathrm{NH}-1$, and the subsequent conformational change brings $\mathrm{N}-13$ closer to the ferryl oxo species. The radical that migrated from $\mathrm{N}-1$ to $\mathrm{C}-4$ reacts with the radical on $\mathrm{N}-13$ to form the $\mathrm{C}-\mathrm{N}$ bond. If the hydrogen bond cannot be abstracted from $\mathrm{O}-13$ or N-13, then the tricyclic compounds are generated via $3 \beta$-hydroxyindolenine formation and ring closure between O-14 and $\mathrm{C}-2$ via epoxidation of the double bond between $\mathrm{C}-1$ and $\mathrm{C}-2$. This study presented beneficial insights on the $\mathrm{P}-450$ catalyzed $\mathrm{C}-\mathrm{O}$ and $\mathrm{C}-\mathrm{C}$ radical coupling reactions in nature, such as those in morphine biosynthesis [26].

\section{Structural analysis of TleC}

Prenylation increases the lipophilicity of a compound, leading to improved bioactivity in many cases. Prenyltransferase (PT) enzymes catalyze prenylation reactions. Their broad substrate specificity and reactivity are quite interesting to researchers, and this enzyme family has been extensively studied $[5,27-31]$. While the C-1 secondary carbocation of the prenyl donor reacts with an acceptor in normal prenylation, the $\mathrm{C}-3$ tertiary carbocation reacts in reverse prenylation, which is rare in nature. TleC catalyzes the reverse prenylation to attach the $\mathrm{C} 10$ geranyl moiety on the C-7 of indolactam V, in the same way as LtxC in lyngbyatoxin A biosynthesis (Fig. 7) [14]. To determine the mechanism of $\mathrm{C} 10$ reverse prenylation, which had not been identified in any studies, we conducted X-ray crystallization studies of $\mathrm{TleC}$ and $\mathrm{MpnD}$ complexed with dimethylallyl-S-thiophosphate and indolactam V, and reported their ABBA-fold structures [32] at 2.10 and $1.40 \AA$ resolutions, respectively. MpnD is a homolog of TleC that transfers a C5 dimethylallyl group on the same position of indolactam V $[33,34]$. TleC and MpnD share similar overall structures and the indolactam $\mathrm{V}$ binding site, but they possess different manners of prenyl chain binding. The $\mathrm{C} 10$ alkyl group of geranyl diphosphate (GPP) is retained in TleC through hydrophobic interactions, contributed by Trp97, Phe170, and Ala173. These residues are, respectively, replaced with Tyr80, Trp157, and Met159 in MpnD (Fig. 7). In TleC, Trp97 is flipped by $70^{\circ}$ when the substrates are bound to the enzymes, and the cavity volume is increased to accept the $\mathrm{C} 10$ alkyl group of GPP. The TleC A173M mutant showed an enhanced preference for dimethylallyl phosphate, and no longer accepted GPP. Interestingly, the TleC W97Y/A173M mutant yielded teleocidin A-2, a C-19 stereoisomer of teleocidin A-1, from indolactam $\mathrm{V}$ and GPP, suggesting the potential of enzyme engineering through this mutation to produce compounds with different stereochemistry. In the TleC complex structure, the distance from C-3 of DMAPP to $\mathrm{C}-7$ of indolactam $\mathrm{V}$ was $3.3 \AA$, and is closer than the distance from $\mathrm{C}-1$ of DMAPP to N-1 of indolactam V (6.0 ̊) (Fig. 7). These data suggested that the direct C-7 
A

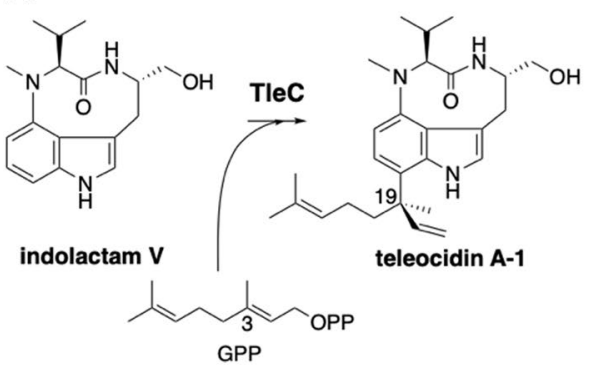

$\overbrace{}^{n^{2}}$

indolactam $\mathbf{V}$
B

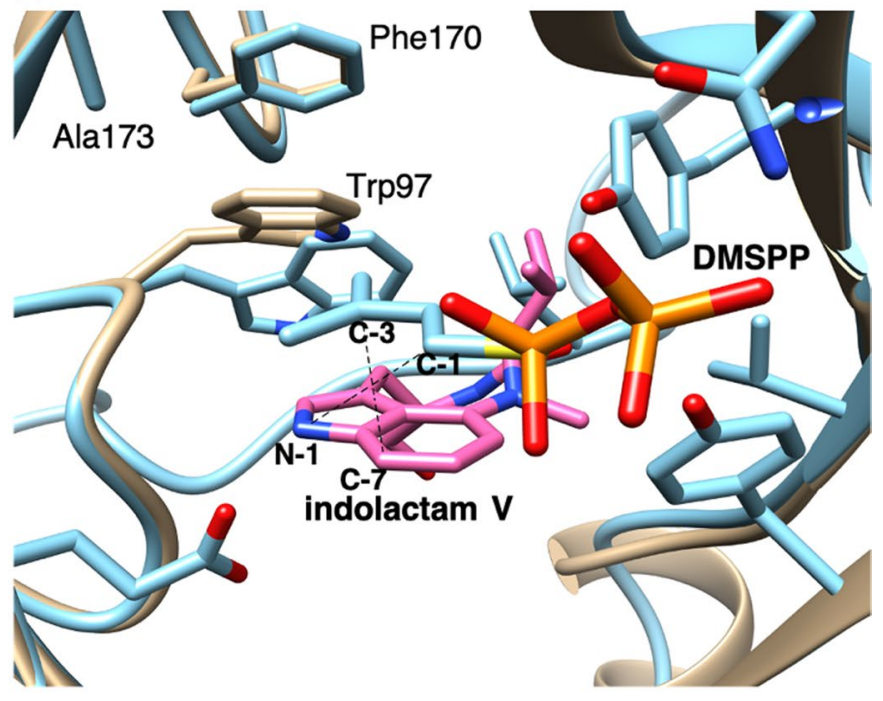

Fig. 7 a The reactions of TleC and MpnD. b The active site structures of TleC. The apo structure (PDB: 4YZK) and the structure complexed with indolactam V and DMSPP (PDB: 4YZL) are colored brown and cyan, respectively

reverse prenylation is more likely than the previously proposed two-step reactions, including $\mathrm{N}-1$ normal prenylation and aza-Claisen rearrangement [12].

\section{Structural analysis of TleD}

As shown above, the terpene cyclization of teleocidin B was triggered by a methyltransferase TleD [18]. TleD belongs to a family of SAM-dependent methyltransferases which
Fig. 8 Active site structure of TleD (PDB: 5GM2). Chains A and $\mathrm{B}$ are colored brown and blue, respectively

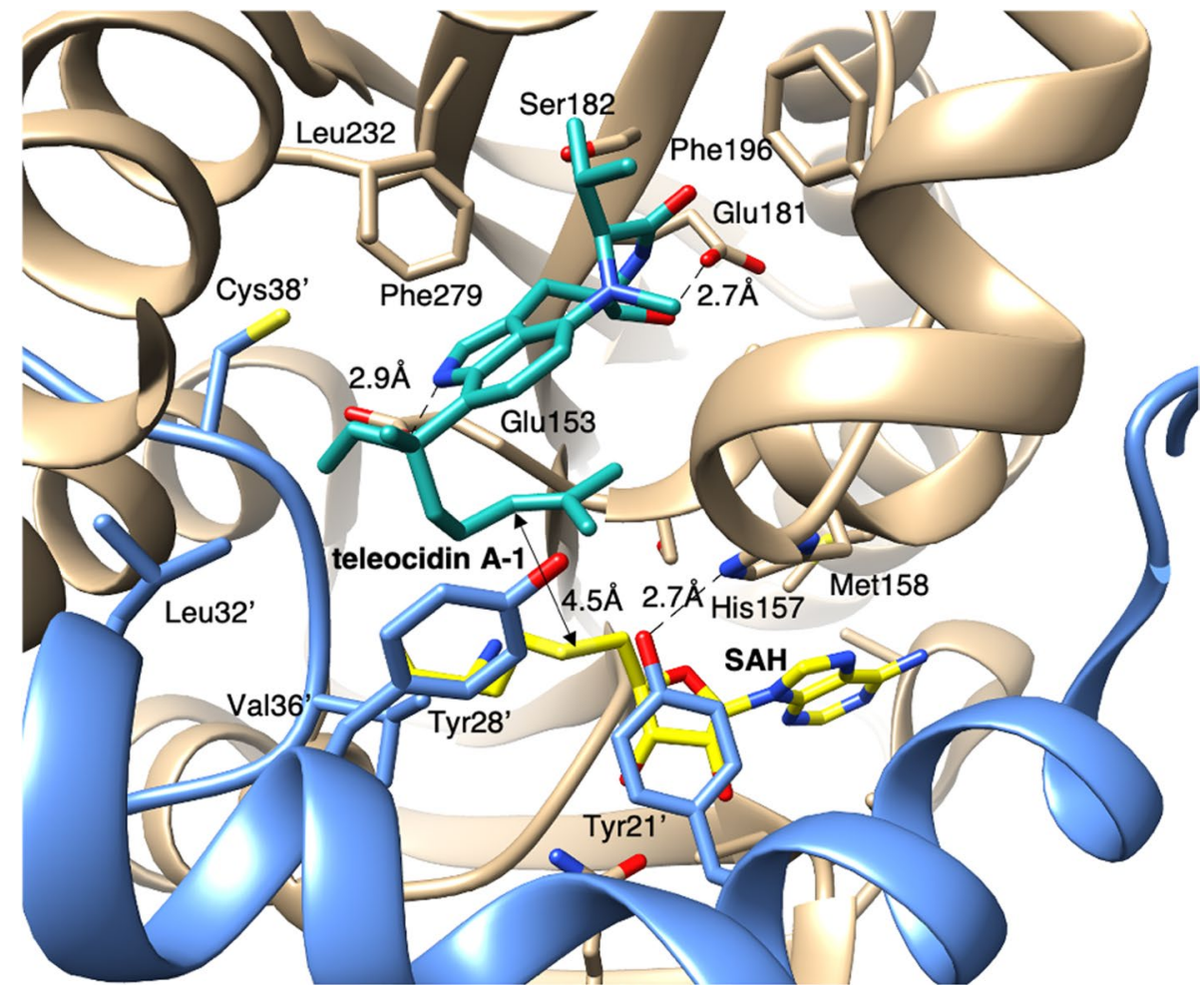


catalyze $\mathrm{C}$-, $\mathrm{O}$-, and $\mathrm{N}$-methylation and affects the polarity of the products [35]. In 2016, Yu et al. reported the $2.80 \AA$ resolution X-ray crystal structure of TleD complexed with $S$-adenosylhomocysteine (SAH) and teleocidin A-1 [36]. The overall structure of its hexamer resembles a typical class I SAM-MT fold [37] with an additional $N$-terminal $\alpha$-helix. The additional $\alpha$-helix penetrates into the core of another monomer, and the active site structure is created between them with assistance from the hydrogen bonding between His 157 (chain A) and Tyr21' (chain B) (Fig. 8). In the active site, SAH is bound via a hydrogen-bond network and van der Waals interactions, and teleocidin A-1 is accommodated by hydrophobic interactions with Tyr21', Tyr28', Leu32', Val36', Cys38', Ser182, Phe196, Leu232, and Phe279 and hydrogen bonds with Glu153 and Glu181 (Fig. 8). The dihedral angle C23-C24-C25-C26 of teleocidin A- 1 is $58^{\circ}$ in this model and is consistent with the $R e$-face stereochemistry to produce teleocidin $\mathrm{B}-4$ and des- $O$-methylolivoretin C. The distance between the S atom of SAH and C25 is $4.5 \AA$, which is reasonable for a $C$-methyltransfer reaction. Their molecular dynamics simulation starting from the one possible conformation showed that the dihedral angle of C23-C24-C25-C26 becomes $60-90^{\circ}$ in 1-ns of simulation, thus also supporting the proposed the conformation of telocidin A-1. The N-terminal $\alpha$-helix is conserved in SpnF, a SAM-dependent methyltransferase-like enzyme that catalyzes [4+2] cycloaddition in spinosyn biosynthesis, and it also helps to create the sealed reaction cavity [38, 39]. The hydrophobic nature of the TleD reaction cavity is beneficial to protect the carbocation from an attack by water. This study provided the first structural information on $C$-methylation-triggered terpene cyclization. However, the proposed structural model lacked information on the mechanism by which TleD facilitates the 1,2-hydride shift and arranges the spiro-intermediate to teleocidin B-4 and des- $O$-methylolivoretin $\mathrm{C}$. A detailed kinetic isotope effect calculation and molecular dynamics study would be useful to investigate the more detailed mechanism, as in SpnF studies [40, 41]. Recently, the C-MT from the rhizobacterium Serratia plymuthica $4 \mathrm{Rx} 13$ was reported to cyclize farnesyl diphosphate to produce pre-sodorifen pyrophosphate [42]. The comparison of this enzyme structure with that of TleD will yield beneficial mechanistic insights.

\section{Conclusion}

This review has summarized the knowledge on the biosynthesis of the terpene indole teleocidin $\mathrm{B}$. The reaction mechanisms, including the $\mathrm{C}-\mathrm{N}$ bond formation by the $\mathrm{P} 450$ oxygenase TleB, the prenyltransfer by $\mathrm{TleC}$, and the terpene cyclization triggered by the methyltransferase TleD, have been discussed in detail, based on the X-ray crystal structures. Currently, their reactions have been analyzed by static structural analyses, but dynamic structural analyses, such as small-angle $\mathrm{X}$-ray scattering, $\mathrm{X}$-ray free-electron laser, and molecular dynamics simulation, would be useful to examine the dynamic structures of these enzymes in the future. Their reaction mechanisms are intriguing from an enzymology viewpoint, and the knowledge is useful for enzyme engineering to create novel medicinal compounds, by using unnatural substrates or heterologous expression systems [43-46]. By complementing the organic synthetic methods [47, 48], more biosynthetic methods to create the analogs will be developed. Among the teleocidin analogs, we will be able to discover useful subtype-specific PKC activators possessing antitumor activities.

Acknowledgement This work was supported by a Grant-in-Aid for Scientific Research from the Ministry of Education, Culture, Sports, Science and Technology, Japan (JSPS KAKENHI Grant Number JP17H04763 and JP19H04641), Mochida Memorial Foundation for Medical and Pharmaceutical Research, Japan Foundation for Applied Enzymology, Kato Memorial Bioscience Foundation, and The Asahi Glass Foundation.

\section{Declarations}

Conflict of interest The author declares no conflict of interest.

Open Access This article is licensed under a Creative Commons Attribution 4.0 International License, which permits use, sharing, adaptation, distribution and reproduction in any medium or format, as long as you give appropriate credit to the original author(s) and the source, provide a link to the Creative Commons licence, and indicate if changes were made. The images or other third party material in this article are included in the article's Creative Commons licence, unless indicated otherwise in a credit line to the material. If material is not included in the article's Creative Commons licence and your intended use is not permitted by statutory regulation or exceeds the permitted use, you will need to obtain permission directly from the copyright holder. To view a copy of this licence, visit http://creativecommons.org/licenses/by/4.0/.

\section{References}

1. O'Connor SE, Maresh JJ (2006) Chemistry and biology of monoterpene indole alkaloid biosynthesis. Nat Prod Rep 23:532-547

2. Marcos IS, Moro RF, Costales I et al (2013) Sesquiterpenyl indoles. Nat Prod Rep 30:1509-1526

3. Xu W, Gavia DJ, Tang Y (2014) Biosynthesis of fungal indole alkaloids. Nat Prod Rep 31:1474-1487

4. Rudolf JD, Alsup TA, Xu B, Li Z (2020) Bacterial terpenome. Nat Prod Rep. Advance Article

5. Awakawa T, Liu X (2020) Biosynthesis of hapalindole type alkaloids. Comprehensive Natural Products III: Chemistry and Biology, vol 2. Elsevier, Amsterdam, pp 486-499

6. Arcoleo JPI, Weinstein B (1985) Activation of protein kinase C by tumor promoting phorbol esters, teleocidin and aplysiatoxin in the absence of added calcium. Carcinogenesis 6:213-217 
7. Takashima M, Sakai H (1960) A new toxic substance, teleocidin, produced by streptomyces: Part II. biological studies of teleocidin. J Agric Chem Soc Japan 24:652-655

8. Takashima M, Sakai H, Mori R, Arima K (1962) A new toxic substance, teleocidin, produced by Streptomyces. Agric Biol Chem 26:660-678

9. Nakata H, Harada H, Hirata Y (1966) The structure of teleocidin B. Tetrahedron Lett 23:2515-2522

10. Sakabe N, Harada H, Hirata Y et al (1966) X-ray structure determination of dihydroteleocidin B monobromoacetate. Tetrahedron Lett 7:2523-2525

11. Hitotsuyanagi Y, Aimi N, Sakai SI et al (1984) Isolation and structure elucidation of teleocidin B-1, B-2, B-3, and B-4. Chem Pharm Bull 32:4233-4236

12. Irie K, Kajiyama S, Funaki A et al (1990) Biosynthesis of indole alkaloid tumor promoters teleocidins (I) Possible biosynthetic pathway of the monoterpenoid moieties of teleocidins. Tetrahedron Lett 46:2773-2788

13. Cardellina JH, Marner FJ, Moore RE (1979) Seaweed dermatitis: Structure of lyngbyatoxin A. Science (80-) 204:193-195

14. Edwards DJ, Gerwick WH (2004) Lyngbyatoxin biosynthesis: Sequence of biosynthetic gene cluster and identification of a novel aromatic prenyltransferase. J Am Chem Soc 126:11432-11433

15. Antal CE, Hudson AM, Kang E et al (2015) Cancer-associated protein kinase $\mathrm{C}$ mutations reveal kinase's role as tumor suppressor. Cell 160:489-502

16. Awakawa T, Abe I (2018) Biosynthesis of the teleocidin-type terpenoid indole alkaloids. Org Biomol Chem 16:4746-4752

17. Abe I (2018) Biosynthetic studies on teleocidins in Streptomyces. J Antibiot (Tokyo) 71:763-768

18. Awakawa T, Zhang L, Wakimoto T et al (2014) A methyltransferase initiates terpene cyclization in teleocidin B biosynthesis. $\mathrm{J}$ Am Chem Soc 136:9910-9913

19. Read JA, Walsh CT (2007) The lyngbyatoxin biosynthetic assembly line: chain release by four-electron reduction of a dipeptidyl thioester to the corresponding alcohol. J Am Chem Soc 129:15762-15763

20. Huynh MU, Elston MC, Hernandez NM et al (2010) Enzymatic production of (-)-indolactam $\mathrm{V}$ by LtxB, a cytochrome $\mathrm{P} 450$ monooxygenase. J Nat Prod 73:71-74

21. Nakagawa Y (2012) Artificial analogs of naturally occurring tumor promoters as biochemical tools and therapeutic leads. Biosci Biotechnol Biochem 76:1262-1274

22. Zhang X, Li S (2017) Expansion of chemical space for natural products by uncommon P450 reactions. Nat Prod Rep 34:1061-1089

23. Walsh CT (2014) Biological matching of chemical reactivity: Pairing indole nucleophilicity with electrophilic isoprenoids. ACS Chem Biol 9:2718-2728

24. He F, Mori T, Morita I et al (2019) Molecular basis for the $\mathrm{P} 450$-catalyzed $\mathrm{C}-\mathrm{N}$ bond formation in indolactam biosynthesis. Nat Chem Biol 15:1206-1213

25. Poulos TL (2014) Heme enzyme structure and function. Chem Rev 114:3919-3962

26. Barton DHR, Lirby GW, Steglich W et al (1965) Investigations on the biosynthesis of morphine alkaloids. J Chem Soc 0:2423-2438

27. Wong CP, Awakawa T, Nakashima Y et al (2018) Two distinct substrate binding modes for the normal and reverse prenylation of hapalindoles by the prenyltransferase AmbP3. Angew Chemie Int Ed 57:560-563

28. Awakawa T, Mori T, Nakashima $Y$ et al (2018) Molecular insight into the $\mathrm{Mg} 2+$-dependent allosteric control of indole prenylation by aromatic prenyltransferase AmbP1. Angew Chemie Int Ed 57:6810-6813

29. Mori T (2020) Enzymatic studies on aromatic prenyltransferases. J Nat Med 74:501-512
30. Li SM (2010) Prenylated indole derivatives from fungi: structure diversity, biological activities, biosynthesis and chemoenzymatic synthesis. Nat Prod Rep 27:57-78

31. Tanner ME (2015) Mechanistic studies on the indole prenyltransferases. Nat Prod Rep 32:88-101

32. Kuzuyama T, Noel JP, Richard SB (2005) Structural basis for the promiscuous biosynthetic prenylation of aromatic natural products. Nature 435:983-987

33. Mori T, Zhang L, Awakawa T et al (2016) Manipulation of prenylation reactions by structure-based engineering of bacterial indolactam prenyltransferases. Nat Commun 7:1-11

34. Ma J, Zuo D, Song Y et al (2012) Characterization of a single gene cluster responsible for methylpendolmycin and pendolmycin biosynthesis in the deep sea bacterium Marinactinospora thermotolerans. ChemBioChem 13:547-552

35. Martin JL, Mcmillan FM (2002) SAM (dependent) I AM: the S-adenosylthionine-dependent methyltransferase fold. Curr Opin Struct Biol 12:783-793

36. Yu F, Li M, Xu C et al (2016) Crystal structure and enantioselectivity of terpene cyclization in SAM-dependent methyltransferase TleD. Biochem J 473:4385-4397

37. Schubert HL, Blumenthal RM, Cheng X (2003) Many paths to methyltransfer: a chronicle of convergence. Trends Biochem Sci 28:329-335

38. Kim HJ, Ruszczycky MW, Choi SH et al (2011) Enzyme-catalysed [4+2] cycloaddition is a key step in the biosynthesis of spinosyn A. Nature 473:109-112

39. Fage CD, Isiorho EA, Liu Y et al (2015) The structure of SpnF, a standalone enzyme that catalyzes [ $4+2]$ cycloaddition. Nat Chem Biol 11:256-258

40. Jeon B-s, Ruszczycky MW, Russell WK et al (2017) Investigation of the mechanism of the SpnF-catalyzed [4+2]-cycloaddition reaction in the biosynthesis of spinosyn A. Proc Natl Acad Sci USA 114:10408-10413

41. Yang Z, Yang S, Yu P et al (2018) Influence of water and enzyme SpnF on the dynamics and energetics of the ambimodal $[6+4] /$ [4+2] cycloaddition. Proc Natl Acad Sci USA 115:E848-E855

42. Von Reuss S, Domik D, Lemfack MC et al (2018) Sodorifen biosynthesis in the Rhizobacterium serratia plymuthica involves methylation and cyclization of MEP-derived farnesyl pyrophosphate by a SAM-dependent C-methyltransferase. J Am Chem Soc 140:11855-11862

43. Morita I, Mori T, Mitsuhashi T et al (2020) Exploiting a C-N bond forming cytochrome $\mathrm{P} 450$ monooxygenase for c-s bond formation. Angew Chem Int Ed Engl 59:3988-3993

44. Morita I, Mori T, Abe I (2020) Enzymatic formation of indolactam scaffold by $\mathrm{C}-\mathrm{N}$ bond-forming cytochrome $\mathrm{P} 450$ oxidases in teleocidin biosynthesis. Chemistry 27:2963-2972

45. Zhang L, Hoshino S, Awakawa T et al (2016) Structural diversification of lyngbyatoxin A by host- dependent heterologous expression of the tleABC biosynthetic gene cluster. ChemBioChem 17:1407-1411

46. Ongley SE, Bian X, Zhang Y et al (2013) High-titer heterologous production in $\mathrm{E}$. coli of lyngbyatoxin, a protein kinase $\mathrm{C}$ activator from an uncultured marine cyanobacterium. ACS Chem Biol 8:1888-1893

47. Gutekunst WR, Baran PS (2011) C-H functionalization logic in total synthesis. Chem Soc Rev 40:1976-1991

48. Nakamura H, Yasui K, Kanda Y et al (2019) 11-Step total synthesis of teleocidin B-1-B-4. J Am Chem Soc 141:1494-1497

Publisher's Note Springer Nature remains neutral with regard to jurisdictional claims in published maps and institutional affiliations. 